\title{
La fotogrametría se convierte en una poderosa herramienta para la conservación y difusión del patrimonio paleontológico
}

\author{
La fotogrametría es una disciplina que permite reconstruir un objeto en tres dimensiones mediante fotografías \\ sacadas desde diferentes ángulos. En los últimos años ha habido un apogeo del estudio de fósiles por \\ medio de visualizaciones digitales tridimensionales. Sus ventajas para la investigación paleontológica son \\ evidentes: se trata de una técnica no invasiva que permite la exploración in situ, a menor coste que otros \\ métodos. Por tanto, la fotogrametría digital está convirtiéndose en una herramienta poderosa y de amplia \\ utilización para la conservación, la divulgación y difusión del patrimonio paleontológico.
}

Ignacio Díaz-Martínez, Paolo Citton, Silvina de Valais | Instituto de Investigación en Paleobiología y Geología, Universidad Nacional de Río Negro-CONICET

Esperanza García-Ortiz | Instituto de Estudios Riojanos

URL de la contribución <www.iaph.es/revistaph/index.php/revistaph/article/view/4191>

La fotogrametría es una disciplina que permite reconstruir un objeto en tres dimensiones (3-D) mediante fotografías sacadas desde diferentes ángulos. Hoy en día, esta técnica aprovecha la capacidad de cálculo de los ordenadores modernos, el relativo bajo coste de las cámaras digitales y la consiguiente capacidad de tomar fotos de alta resolución.

En paleontología, tradicionalmente los fósiles se han figurado mediante dibujos o fotografías. No obstante, en los últimos años ha habido un apogeo de la "paleontología virtual", es decir, el estudio de fósiles por medio de visualizaciones digitales tridimensionales (3-D) o fósiles virtuales (véase SUTTON; RAHMAN; GARWOOD, 2016: 1-20, para más información). La mayor parte de las veces, estos modelos se han obtenido gracias a la fotogrametría, tomografía o láser escáner. Uno de los beneficios que presenta la fotogrametría respecto a otros métodos de digitalización es que no hace falta mover el fósil del lugar donde esté, ya sea en el campo o depositado en una colección, tal como sucede con la tomografía. Por otro lado, es un método no invasivo, donde únicamente se sacan fotografías y no se ejerce ningún contacto con el fósil. Asimismo, tiene un coste más bajo que el láser escáner y que el de un tomógrafo, ya que se puede trabajar con programas informáticos gratuitos y con dispositivos de uso doméstico (e.g., cámaras digitales compactas, teléfonos móviles). Otra cualidad de la presente técnica se refiere a la posibilidad de alcanzar un gran realismo al texturizar el modelo 3-D obtenido con las propias fotografías utilizadas. Finalmente, cabe destacar que el resultado (la generación del modelo 3-D) es casi inmediato y únicamente depende de la capacidad de cada ordenador para procesar los datos. Gracias a los beneficios que sustenta, el interés por la generación de modelos 3-D en paleontología se ha incrementado recientemente. La mayor parte de las veces dichos modelos se han usado con fines meramente científicos. No obstante, también se ha considerado su utilización con otros fines, tales como orientados hacia la gestión del patrimonio (REMONDINO, 2011: 1104-1138; CAYLA; HOBLÉA; REYNARD, 2014: 89-90; FERNÁNDEZLOZANO; GUTIÉRREZ-ALONSO, 2017: 163-173), siendo empleados en su conservación, divulgación y uso turístico.

El patrimonio paleontológico está integrado por la suma de colecciones, ejemplares, museos y exposiciones, que reúnen el material utilizado en investigación, enseñanza o divulgación (i.e., patrimonio mueble) y por el conjunto de yacimientos conocidos (i.e. patrimonio inmueble) (CARCAVILLA, 2014: 5-17). La fotogrametría es una herramienta que se puede usar en ambos tipos de patrimonio adaptándose a las particularidades de cada 


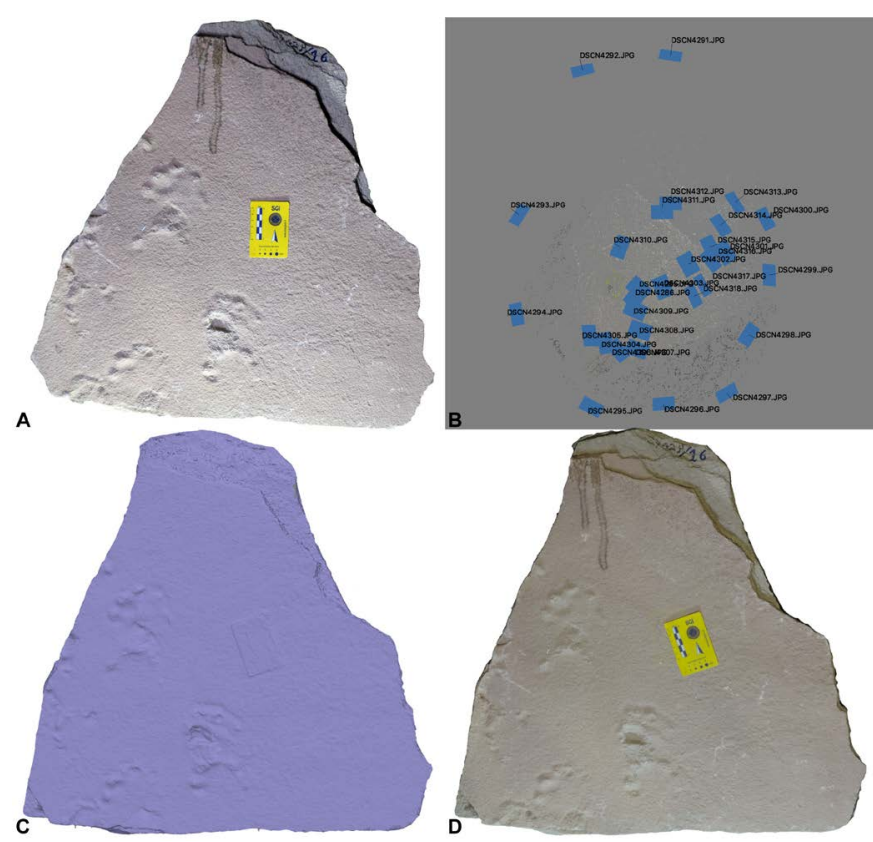

Ejemplo de procesado fotogramétrico de una laja con huellas de tetrápodos. A. Fotografía de la laja MPCA 27029-16, conservada en el Museo Provincial Carlos Ameghino, Cipolletti, provincia de Río Negro, Argentina. B. Nube de puntos en común obtenida por el programa y basada en el alineamiento de las fotografías. C. Sólido obtenido a partir de una malla calculada desde la nube de puntos. D. Modelo con textura de la laja real | modelo fotogramétrico Agisoft Photoscan Pro; fotografía y modelos Paolo Citton

uno de ellos (e. g., tamaño, ubicación, complejidad, iluminación). Es muy común su utilización en yacimientos de gran tamaño, como por ejemplo en yacimientos de huellas de vertebrados fósiles (e.g., DÍAZ-MARTÍNEZ; GARCÍA-ORTIZ; PÉREZ-LORENTE, 2015: 167-175; CITTON; ROMANO; CARLUCCIO et ál., 2017: 47-59). Mayoritariamente, estos yacimientos se encuentran a la intemperie y expuestos a los agentes atmosféricos y a riesgos antrópicos (GARCÍA-ORTIZ; FUERTESGUTIÉRREZ; FERNÁNDEZ-MARTÍNEZ, 2014: 463479; GARCÍA ORTIZ DE LANDALUCE, 2017).

El uso de la fotogrametría permite realizar un control de su conservación mediante la superposición de modelos 3-D periódicos para observar si han sufrido algún daño con el paso del tiempo. Con respecto al patrimonio paleontológico mueble, la fotogrametría aporta numerosos usos y ventajas. Por ejemplo, sería posible tener una base de datos digital de las colecciones, salvaguardándolas de cara a futuras investigaciones. Asimismo, se podrían analizar desde cualquier lugar del mundo sin
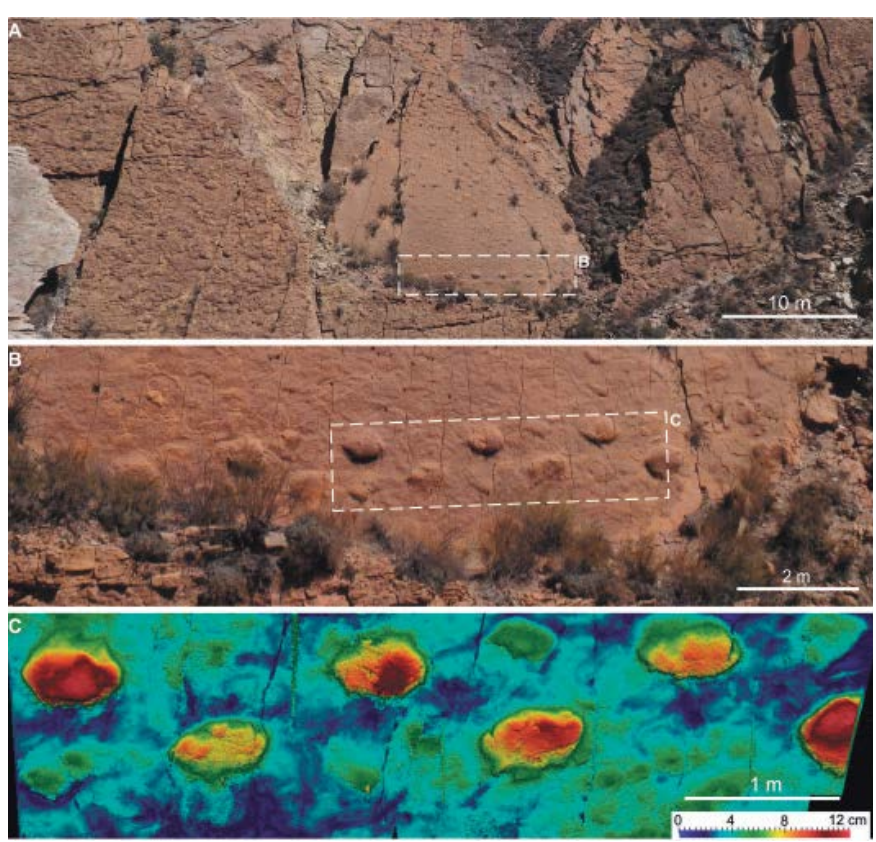

Modelo de elevación digital de un yacimiento icnológico. A. Fotografía general del yacimiento icnológico del valle del Tonco en Jujuy, Argentina (el estrato con huellas está invertido). B. Detalle de una rastrillada de un dinosaurio saurópodo. C. Modelo de elevación calculado a partir de un modelo fotogramétrico de parte de la rastrillada (los colores representan diferentes alturas) | modelo fotogramétrico Agisoft Photoscan Pro, modelo de elevación ParaView;

fotografías Carlos Cónsole-Gonellas; modelo Ignacio Díaz-Martínez

tener que trasladarse a la colección, ni tener que manipularlos. Incluso en el caso de que el fósil haya sufrido algún desperfecto, es posible hacer una copia con una impresora 3-D.

De esta forma, la fotogrametría digital está convirtiéndose en una herramienta poderosa y de amplia utilización para la conservación, la divulgación y difusión del patrimonio paleontológico, no solo en el campo científico. Estas posibilidades también se ven facilitadas por la existencia de plataformas en línea que actúan como repositorios digitales (e.g., MorphoMuseuM, MorphoSource) que almacenan tanto el material electrónico como la autoría de la digitalización. La técnica, por lo tanto, se torna prioritaria para aquellos elementos que se encuentran expuestos a agentes de deterioro que incrementan su riesgo de degradación, y permite conservar su información para el beneficio de las generaciones futuras. 


\section{BIBLIOGRAFÍA}

- CARCAVILlA, L. (2014) Guía práctica para entender el patrimonio geológico. Enseñanza de las Ciencias de la Tierra, 22, 2014, pp. 5-17

- CAYLA, N.; HOBLÉA, F.; REYNARD, E. (2014) New Digital Technologies Applied to the Management of Geoheritage. Geoheritage, 6 (2), 2014, pp. 89-90

- CITTON, P.; ROMANO, M.; CARLUCCIO, R.; D'AJELLO CARACCIOLO, F.; NICOLOSI, I.; NICOSIA, U.; SACCHI, E.; SPERANZA, G.; SPERANZA, F. (2017) The first dinosaur tracksite from Abruzzi (Monte Cagno, Central Apennines, Italy). Cretaceous Research, 73, 2017, pp. 47-59

- DÍAZ-MARTÍNEZ, I.; GARCÍA-ORTIZ, E.; PÉREZLORENTE, F. (2015) A new dinosaur tracksite with small footprints in the Urbión Group (Cameros Basin, Lower Cretaceous, La Rioja, Spain). Journal of Iberian Geology, 41 (1), 2015, pp. 167-175

- FERNÁNDEZ-LOZANO, J.; GUTIÉRREZ-ALONSO, G. (2017) The Alejico Carboniferous Forest: a 3D-Terrestrial and UAV-Assisted Photogrammetric Model for Geologic Heritage Preservation. Geoheritage, 9 (2), 2017, pp. 163-173

- GARCÍA-ORTIZ, E.; FUERTES-GUTIÉRREZ, I.; FERNÁNDEZ-MARTÍNEZ, E. (2014) Concepts and terminology for the risk of degradation of geological heritage sites: fragility and natural vulnerability, a case study. Proceedings of the Geologists' Association, 125, 2014, pp. 463-479

- garcía ortiz dE LANDALUCE, E. (2017) Análisis de los yacimientos de icnitas de dinosaurios de La Rioja ( $N$ de España) como recurso patrimonial y aplicación de nuevas tecnologías a su estudio. CD-ROM. Memoria de Tesis Doctoral. León: Universidad, Área de Publicaciones, 2017 (Serie TESIS DOCTORALES)

- REMONDINO, F. (2011) Heritage recording and 3D modeling with photogrammetry and 3D scanning. Remote Sensing, 3(6), 2011, pp. 1104-1138

- SUTTON, M.; RAHMAN, I.; GARWOOD, R. (2016) Virtual paleontology-An overview. The Paleontological Society Papers, 22, 2016, pp. 1-20 